\title{
Coulometrische Festelektrolytsensoren - Anwendungen und Fehlerquellen
}

\author{
J. Zosel ${ }^{1}$, M. Schelter ${ }^{1}$, V. Vashook ${ }^{1}$, U. Guth ${ }^{2}$, M. Mertig ${ }^{1,2}$ \\ ${ }^{1}$ Kurt-Schwabe-Institut für Mess- und Sensortechnik e.V. Meinsberg, \\ Kurt-Schwabe-Straße 4, D-04736 Waldheim, Deutschland \\ zosel@ksi-meinsberg.de \\ 2 Technische Universität Dresden, Professur für Physikalische Chemie, Mess- und Sensortechnik, \\ D-01062 Dresden, Deutschland
}

\begin{abstract}
Kurzfassung:
Coulometrische Festelektrolytsensoren werden in zunehmendem Maß für verschiedene Applikationen, z.B. für die Sauerstoffmessung und die Forschung an keramischen Materialien eingesetzt. Einer ihrer wesentlichen Vorteile betrifft ihre relativ genaue Signalabhängigkeit vom Faradayschen Gesetz, die einen kalibrierfreien Betrieb über einen langen Zeitraum ermöglicht. Der Einsatz dieser Sensoren zur Messung von Spurenkonzentrationen im ppm- und ppb-Bereich offenbart Abweichungen vom Faradayschen Gesetz, die durch die elektronische Leitfähigkeit des Elektrolyts (statisch) sowie durch Potential- und Temperaturänderungen oder elektromagnetische Einstreuungen (dynamisch) verursacht werden. Die Ergebnisse belegen, dass die bei Potentialen zwischen -900 und $-350 \mathrm{mV}$ gegen eine $\mathrm{Pt} /$ Luft-Referenzelektrode gefundenen statischen Abweichungen vollständig durch die Defektelektronenleitfähigkeit verursacht werden. Die bei Potentialen unter $-1400 \mathrm{mV}$ gemessenen Abweichungen beruhen im Wesentlichen auf der Elektronenleitfähigkeit des Festelektrolyts und sind deutlich niedriger als vergleichbare Literaturangaben. Der an einer Rohrzelle mit $6 \mathrm{~mm}$ Außendurchmesser, $1 \mathrm{~mm}$ Elektrolytdicke und $60 \mathrm{~mm}$ Länge gemessene Ladungstransfer nach einem Potentialsprung um $-50 \mathrm{mV}$ beträgt 0,45 - $310 \mathrm{mAs}$. Diese z.T. relativ hohen Werte, die oft den während einer coulometrischen Bestimmung aufzulösenden Messwert deutlich übersteigen, unterstreichen die Wichtigkeit der präzisen Potentialkontrolle bei coulometrischen Messungen. Durch die Berücksichtigung dieser Abweichungen konnte demonstriert werden, dass coulometrische Festelektrolyt-Detektoren für die gaschromatographische Analytik im ppb-Bereich erfolgreich eingesetzt werden können.
\end{abstract}

Key words: Coulometrischer Festelektrolytsensor, Sub-ppm-Konzentrationsmessung, Sauerstoffspeicherung im Elektrolyt

\section{Einführung}

Der zunehmende Einsatz coulometrischer Festelektrolytsensoren und Messsysteme in verschiedenen Applikationen [1] beruht auf ihrer relativ genauen Signaleinstellung auf der Basis des Faradayschen Gesetzes, die einen kalibrierfreien Betrieb über Zeiträume von mehreren Jahren und Messbereiche von mehr als 5 Zehnerpotenzen ermöglichen. Beim Einsatz solcher Sensoren für die Bestimmung von Spurengaskonzentrationen im ppm- und ppb-Bereich werden Abweichungen vom Faradayschen Gesetz sichtbar [2]. Diese Abweichungen sind statischer und dynamischer Natur und bewirken systematische bzw. zufällige Fehler. Während sich die systematischen Fehler weitgehend rechnerisch korrigieren lassen [3], können durch Rauscheinflüsse oder Temperaturschwankungen hervorgerufene zufällige Fehler nur durch Designoptimierungen an Sensoren und Messwandlern bzw. längere Messzeiten verringert werden [4]. Im Beitrag werden Ergebnisse statischer und dynamischer Abweichungen vom Faradayschen Gesetz vorgestellt, die an coulometrischen Sensoren mit einem Festelektrolyt aus stabilisiertem Zirkoniumdioxid auftreten können. Die Ursachen dieser Abweichungen werden diskutiert und Möglichkeiten zu ihrer Verringerung aufgezeigt. Die dadurch erreichbare Absenkung der unteren Messgrenze solcher Systeme wird am Beispiel des Einsatzes als Detektor für die Gaschromatographie beschrieben [5].

\section{Methoden und Materialien}

Für die Experimente wurden rohrförmige aus $8 \mathrm{Mol} .-\%$ Yttriumoxid-stabilisiertem Zirkoniumdioxid (YSZ) als $\mathrm{O}^{2-}$-Ionen-leitendem Festelektrolyt gefertigte Sensoren verwendet (Bild 1). 
Die Durchströmung des einseitig geschlossenen YSZ-Rohres wurde durch eine eingeschobene Kapillare aus Aluminiumoxid gewährleistet. Die Elektroden waren aus Pt-Netzen gefertigt, die auf beiden Gasseiten aufgesintert waren. Die Dimensionen der Durchflusssensoren betrugen $4 \mathrm{~mm}$ Innendurchmesser, $6 \mathrm{~mm}$ Außendurchmesser und $60 \mathrm{~mm}$ Elektrodenlänge. Die Sensoren wurden bei verschiedenen Volumenströmen im Temperaturbereich 650 - 800 ${ }^{\circ} \mathrm{C}$ sowie im Potentialbereich -1500 bis $-300 \mathrm{mV}$ gegen eine $\mathrm{Pt} / \mathrm{Luft}-$ Referenzelektrode im Hinblick auf ihren Signal-strom bei verschwindender Analytkonzentration untersucht. Weiterhin wurde der Sauerstoff-austausch des Sensors im Partialdruckbereich $\mathrm{p}\left(\mathrm{O}_{2}\right)=4,2 \cdot 10^{-17}$ $2,5 \cdot 10^{-3} \mathrm{~Pa}$ nach Potentialschritten um $50 \mathrm{mV}$ durch die Messung der Stromrelaxation über Zeiträume von bis zu 5 h untersucht. Der für die Messungen eingesetzte Stickstoff wurde vor der Durchströmung des Sensors im Bereich 10 - $50 \mathrm{ml} / \mathrm{min}$ mit dem Gasreiniger OM-750 (pure gas products) so gereinigt, dass dessen Sauerstoff- und Wasserdampfkonzentration geringer als 2 bzw. als 10 Vol.-ppb waren. Die elektrochemischen Messungen wurden mit einem Potentiostat REF600 (Gamry Instruments) durchgeführt.

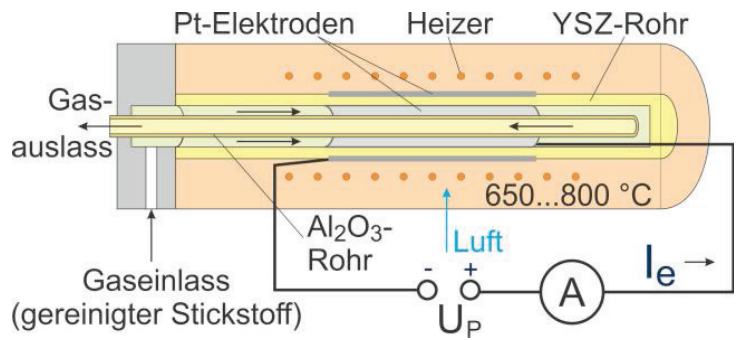

Bild 1. Coulometrischer Durchflusssensor, Dimensionen: Innendurchmesser $=4 \mathrm{~mm}$, Außendurchmesser $=6 \mathrm{~mm}$, Elektrodenlänge $=60 \mathrm{~mm}$.

Die auf diese Weise charakterisierten Sensoren wurden als Detektoren für die Gaschromatographie im Hinblick auf die damit erreichbare untere Nachweisgrenze erprobt. Unter Einsatz des in [6] beschriebenen Gaschromatographen wurden Gasproben, die definierte Spurenkonzentrationen von Methan und Wasserstoff im ppb-Bereich enthielten, des Volumens $1 \mathrm{ml}$ bei Standardbedingungen mit einer gepackten Molsiebsäule bei $50{ }^{\circ} \mathrm{C}$ im Trägergas Argon aufgetrennt und anschließend coulometrisch titriert.

\section{Ergebnisse}

\section{A) Statische Abweichungen}

Die statischen Gleichströme durch die Festelektrolytzelle gemäß Bild 1 bei Durchströmung mit gereinigtem $\mathrm{N}_{2}$ sind für verschiedene Potentiale und Temperaturen in Bild 2 aufgeführt.
Geringfügige Stromänderungen, die durch Spuren von oxidierbaren Gasen im Stickstoff mit Konzentrationen $<50$ Vol.-ppb hervorgerufen werden, wenn der Volumenstrom variiert wird, wurden als Korrektur bei den Resultaten in Bild 2 nach der in [5] beschriebenen Methodik bereits berücksichtigt. Die in Bild 2 gezeigten Kurven weisen im Potentialbereich -350 bis $-850 \mathrm{mV}$ den für YSZ bekannten Verlauf [5, 7] auf, in dem die durch extrinsische Dotierung erzeugte Defektelektronenleitfähigkeit den sich bei blockierter Elektrode kaum ändernden Zellstrom dominiert.

Im Bereich zwischen -850 und $-1300 \mathrm{mV}$ steigt die Elektronenleitfähigkeit an, die dann unter $-1400 \mathrm{mV}$ den Zellstrom weitgehend bestimmt. In diesem Übergangsbereich zwischen -850 und $-1300 \mathrm{mV}$ wurde an der relativ großen Zelle ein ungewöhnlich verlaufender Stromanstieg beobachtet, wenn der Potentialsprung zu Beginn des Experimentes zu niedrigeren Potentialen gerichtet war. Wenn die Messdauer nach dem Sprung von $1 \mathrm{~h}$ auf $5 \mathrm{~h}$ erhöht wird, geht dieser ungewöhnliche Anstieg zwar zurück, verschwindet aber nicht vollständig. Es wird vermutet, dass dieser Verlauf durch langsam reagierende und eventuell auch diffundierende redoxaktive Verunreinigungen im Festelektrolyt verursacht wird.

Die beim niedrigsten untersuchten Potential von -1500 mV auftretenden Sauerstoffpartialdrücke $3,6 \cdot 10^{-29} \mathrm{~Pa}\left(650{ }^{\circ} \mathrm{C}\right)$ bzw. $1,4 \cdot 10^{-24} \mathrm{~Pa}$ $\left(800{ }^{\circ} \mathrm{C}\right)$ führen $\mathrm{Zu}$ einem exponentiellen Stromanstieg gemäß der ansteigenden Elektronenleitfähigkeit [8]. In Bild 3 sind die bei blockierender Elektrode gemessenen Werte für die Zellströme gemeinsam mit Verläufen aufgeführt, die anhand der in [3] publizierten Daten für die Defektelektronen-Leitfähigkeit (a) und die Elektronen-Leitfähigkeit (b) berechnet werden können.

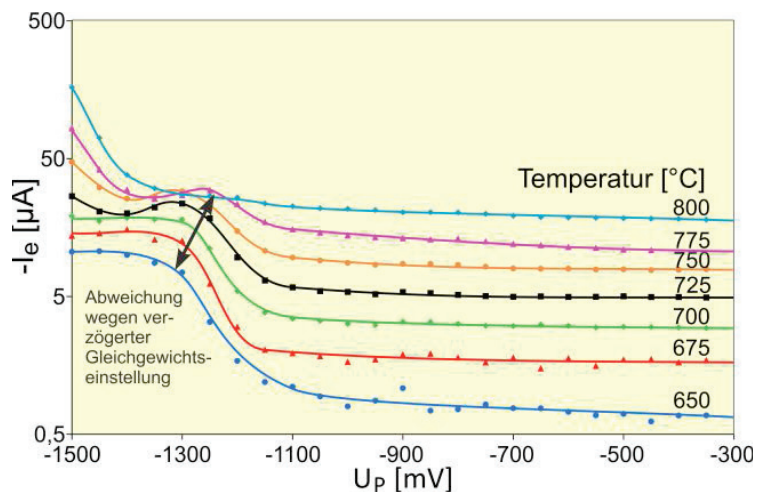

Bild 2. Statische durch Elektronen und Defektelektroden getragene Ströme im coulometrischen Sensor gemäß Bild 1 in gereinigtem Stickstoff-Gasfluss bei verschiedenen Temperaturen und Potentialen. 


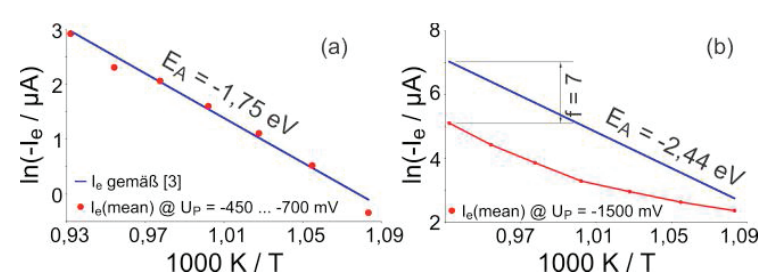

Bild 3. Arrhenius-Graphen des in Bild 2 dargestellten Reststroms bei ausgewählten Potentialen und Vergleich mit den in [3] angegeben Daten der elektronischen Leitfähigkeit

Der im Arrhenius-Plot über der Temperatur aufgetragene Verlauf des Zellstroms belegt, dass im Potentialbereich -450 bis $-700 \mathrm{mV}$ der Zellstrom den Literaturdaten im Rahmen der erreichten Messgenauigkeit folgt, während der bei $-1500 \mathrm{mV}$ und $800{ }^{\circ} \mathrm{C}$ gefundene Zellstrom etwa 7 fach niedriger als der berechnete Strom ist. Die Ursache für dieses Verhalten wird in deutlich geringeren Konzentrationen von Verunreinigungen im hier verwendeten YSZ vermutet, die bei diesen Sauerstoffpartialdrücken Elektronen an das Leitungsband abgeben können.

\section{B) Dynamische Abweichungen}

Typische Zellstromverläufe während und nach einem Potentialsprung sind für verschiedene Startpotentiale und Sprungrichtungen in Bild 4 gezeigt. Diese Stromverläufe wurden durch Peakhöhe und -fläche charakterisiert, wobei die Peakfläche eine Ladungsmenge darstellt, die mit einer durch das Faradaysche Gesetz bestimmten Menge Sauerstoff korrespondiert, der beim Potentialsprung zwischen dem Elektrolyt und der Elektrodenoberfläche einerseits und der Umgebung andererseits ausgetauscht wird. Die Aufzeichnung des Zellstroms erfolgte mit abnehmender Messfrequenz, beginnend mit $10 \mathrm{kHz}$ in den ersten $3 \mathrm{~s}$ nach dem Sprung, gefolgt von $5 \mathrm{~Hz}$ bis $100 \mathrm{~s}$ nach dem Sprung und $0,2 \mathrm{~Hz}$ bis zum Ende der Messung. Die Ergebnisse zeigen eine deutliche Abhängigkeit des Stromverlaufs nach dem Sprung von dessen Richtung und vom Startpotential.

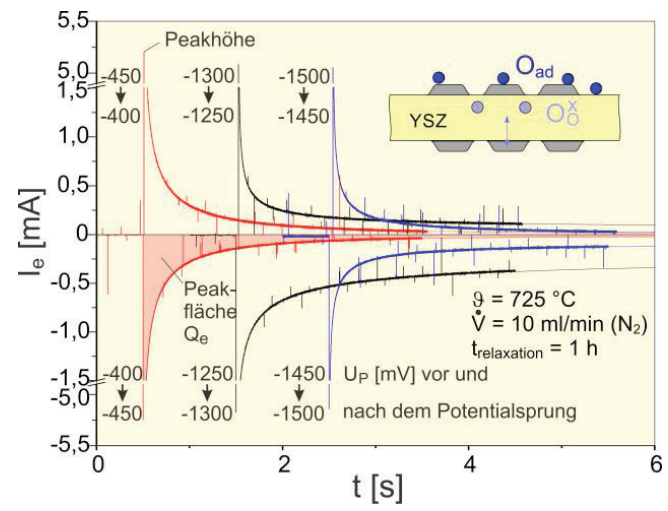

Bild 4. Zellstrom $l_{e}$ während sprungartiger Änderungen der Zellspannung $U_{P}$ von $50 \mathrm{mV}$ in beide Richtungen, coulometrischen Sensor.

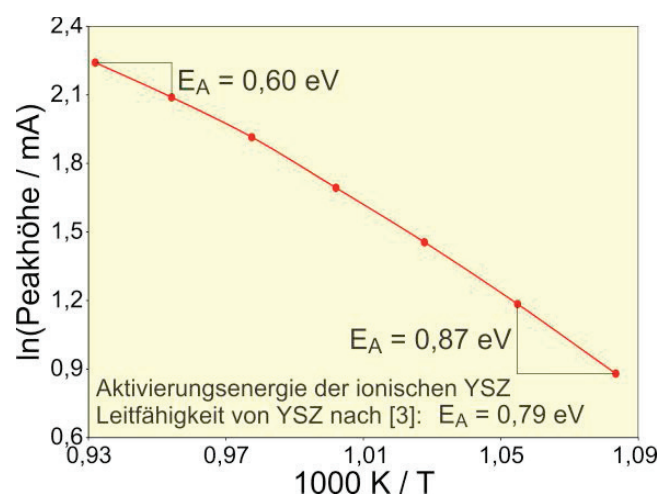

Bild 5. Arrhenius-Graph der Peakhöhe des Zellstroms $l_{e}$ gemäß Bild 4 nach einem Potentialsprung und Vergleich mit der in [3] angegebenen Aktivierungsenergie für die ionische Leitfähigkeit von YSZ.

Ein Vergleich zwischen den drei Kurven der Sprünge zu niedrigeren Potentialen zeigt, dass die Abhängigkeit des Stroms zu einem bestimmten Zeitpunkt t nach dem Potentialsprung keinem eindeutigen Trend im Hinblick auf das Startpotential folgt. Jeder der in Bild 4 gezeigten Strompeaks tritt $0,5 \mathrm{~s}$ nach dem Start der Messung ein. Die im Bild gewählte Zeitdifferenz von $1 \mathrm{~s}$ zwischen den drei Peaks ermöglicht eine angemessene Sichtbarkeit aller Kurven.

Ein genauerer Vergleich zwischen den einzelnen Peakhöhen und -flächen der in Bild 4 als Beispiele dargestellten Stromverläufe nach Potentialsprüngen ist in den Bildern 5 und 6 aufgeführt. Wie erwartet, hängt die Peakhöhe nur von der Temperatur (und der Höhe des Potentialsprungs, die hier jedoch nicht variiert wurde) nicht aber vom Startpotential selbst ab.

Außerdem nimmt die Aktivierungsenergie der Temperaturabhängigkeit des Peakstroms einen Wert an, der dem der ionischen Leitfähigkeit von YSZ sehr nahe kommt. Der initiale Strom nach dem Potentialsprung, der im Wesentlichen zur Beladung der Doppelschichtkapazität dient, wird demnach ausschließlich vom Elektrolytwiderstand limitiert.

Die in Bild 6 gezeigten Verläufe der Peakfläche nach einem Potentialsprung $\mathrm{zu}$ niedrigeren Potentialen weisen sowohl hinsichtlich der Temperatur als auch in Bezug auf das Startpotential verschiedene Trends auf und legen die Vermutung nahe, dass verschiedene Prozesse der Sauerstofffreisetzung bei unterschiedlichen Potentialen dominieren. Diese hängen mit den bereits genannten, redoxaktiven Verunreinigungen im Elektrolyt, mit wechselnden $\mathrm{O}_{2}$-Belegungen der Elektrodenoberfläche sowie mit dem Gaswechsel in geschlossenen Poren im Sinterkörper an der Elektrode zusammen. Der Sauerstoffaustausch in solchen Poren ist vor allem bei den in diesem Experiment gewählten niedrigeren Temperaturen deutlich verzögert. 


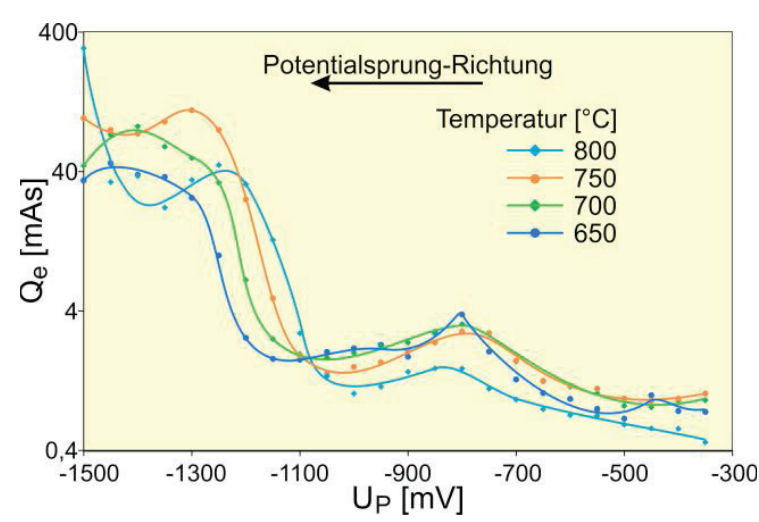

Bild 6. Verlauf der Ladungsmenge $Q_{e}$ (Peakfläche gemäß Bild 4) am coulometrischen Sensor gemäß Bild 1 in Abhängigkeit von der Zellspannung $U_{P}$ vor dem Potentialsprung, die einer Sauerstofffreisetzung bei Potentialerniedrigung um $50 \mathrm{mV}$ entspricht.

Generell lassen sich in den Messkurven folgende zwei Bereiche unterscheiden. Im Potentialbereich zwischen -300 und $-1000 \mathrm{mV}$ werden vergleichsweise kleine Ladungsmengen vermutlich aus Poren und von Elektrodenoberflächen freigesetzt. Bei Potentialen im Bereich unter $-1000 \mathrm{mV}$ beginnt die Sauerstofffreisetzung aus dem Gitter des Festelektrolytes, wodurch die elektronische Leitfähigkeit ansteigt. Diese Freisetzung erreicht zwischen -1300 und $-1400 \mathrm{mV}$ ein erstes Plateau und stammt daher wahrscheinlich im Wesentlichen von Verunreinigungen im Elektrolyt.

Die für die Peakfläche gemessenen Werte differieren untereinander um bis zu vier Zehnerpotenzen im Bereich 0,45 bis 310 mAs. Diese Werte wurden an Sensoren gefunden, deren Abmessungen denen kommerziell erhältlicher coulometrischer Zellen sehr ähnlich sind, und die z.B. für Sauerstoffaustauschmessungen an oxidischen Materialien im Bereich von wenigen pmol eingesetzt werden (10 pmol $\mathrm{O}_{2} \triangleq 3,86$ $\mu \mathrm{As})$. Die bei Potentialänderung am Sensor ausgetauschte Menge an Sauerstoff kann also bei der angestrebten Auflösung deutlich höher sein, als die im Sensoreinsatz zu messende Sauerstoffmenge. Daher ist eine hochpräzise Kontrolle des Elektrodenpotentials während einer coulometrischen Messung unerlässlich.

Im Gegensatz zur langen Zeitdauer, die der Zellstrom nach einem Potentialsprung zur Relaxation benötigt, liegt das Ansprechverhalten coulometrischer Sensoren ähnlich wie bei potentiometrischen Festelektrolytsensoren im Bereich weniger Millisekunden.

\section{C) Applikation als Detektor für die Chromatographie}

Bei Berücksichtigung der in den Abschnitten A) und $B$ ) genannten statischen und dynamischen Abweichungen coulometrischer Sensoren las- sen sich diese als vergleichsweise hochpräzise chromatographische Detektoren zum Nachweis von Spurengasen einsetzen. Mit einem coulometrischen Detektor gemäß Bild 1 wurden solche Untersuchungen durchgeführt und die in Bild 7 gezeigten Ergebnisse erzielt. Die Flächen unter den $\mathrm{CH}_{4}$ - und $\mathrm{H}_{2}$-Peaks können als Ladungsmengen über das Faradaysche Gesetz direkt in die entsprechende Stoffmenge umgerechnet werden, während die Peakhöhe ein Maß für die Konzentration bildet, das von der verwendeten Trennsäule und den Versuchsbedingungen erheblich beeinflusst wird.

Aus den in Bild 7 dargestellten Signalkurven wurden durch Mittelung die in Bild 8 aufgeführten Kalibrierkurven $\mathrm{A}(\mathrm{H})=\mathrm{f}(\mathrm{c})$ (mit $\mathrm{c}=$ Konzentration von $\mathrm{H}_{2}$ bzw. $\mathrm{CH}_{4}$ ) berechnet, aus denen gemäß der IUPAC-Empfehlung die untere Nachweisgrenze berechnet wurde.

Die Werte für diese untere Nachweisgrenze sind als gestrichelte Linien in Bild 8 aufgeführt und liegen mit Ausnahme des Wertes für die Peakfläche bei der $\mathrm{H}_{2}$-Bestimmung immer deutlich niedriger als 100 Vol.-ppb. Somit konnte erstmals der Nachweis erbracht werden, dass coulometrische Festelektrolytsensoren für kalibrierfreie Messungen von Gaskonzentrationen unter 200 Vol.-ppb langzeitstabil eingesetzt werden können.
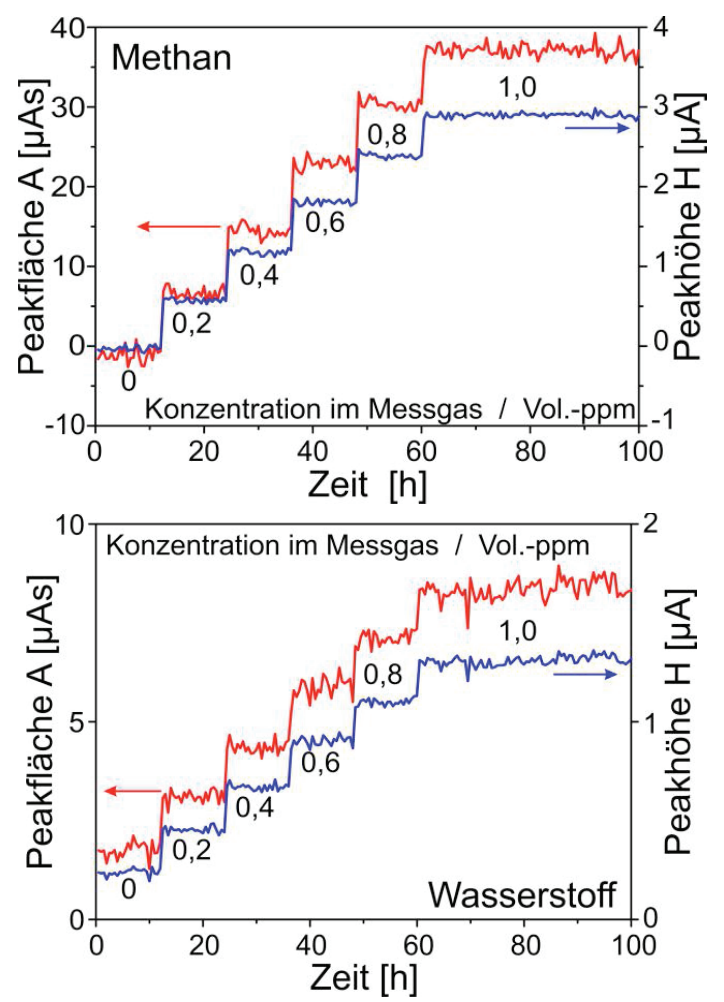

Bild 7: Verlauf der mit einem coulometrischen Detektor gemäß Bild 1 an Modellgas-Proben ermittelten Peakflächen und -höhen bei Konzentrationen von 0 bis 1 Vol.-ppm für Methan und Wasserstoff, Detektortemperatur $650^{\circ} \mathrm{C}$. 

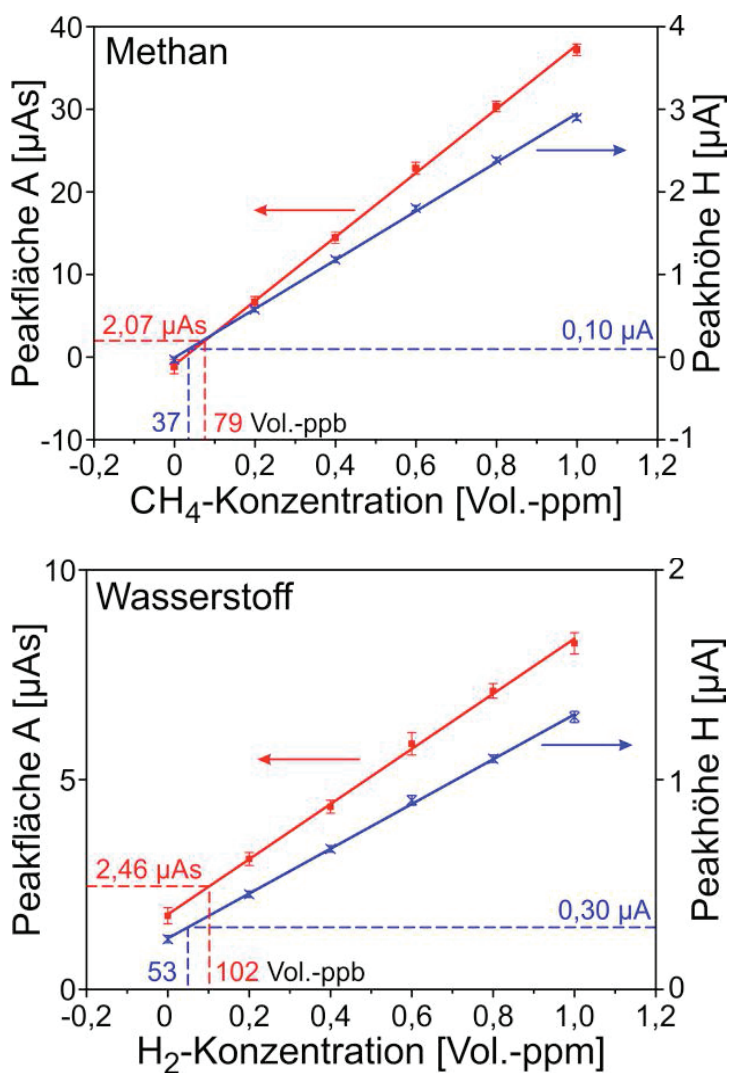

Bild 8: Kalibrierung der in Bild 7 dargestellten Peakflächen und -höhen bei Konzentrationen von 0 bis $1 \mathrm{Vol}$-ppm für $\mathrm{H}_{2}$ und $\mathrm{CH}_{4}$; die Fehlerbalken entsprechen den Standardabweichungen s; die Nachweisgrenzen sind jeweils für $A$ und $H$ angegeben.

\section{Zusammenfassung}

Coulometrische Durchfluss-Sensoren mit stabilisiertem Zirkoniumdioxid als Festelektrolyt für die Sauerstofftitration wurden im Hinblick auf ihre Abweichungen vom Faradayschen Gesetz untersucht. Die Messungen belegen, dass bei konstanter Temperatur und konstantem Elektrodenpotential Abweichungen des Zellstroms vom Faradayschen Gesetz auftreten.

Bei Potentialen zwischen -350 und $-900 \mathrm{mV}$ gegen eine Pt/Luft-Referenzelektrode können diese Abweichungen im Rahmen der erzielbaren Messgenauigkeit vollständig auf die Defektelektronenleitfähigkeit des Festelektrolytes zurückgeführt und somit korrigiert werden.

Bei Potentialen unter -1400 mV und Sauerstoffpartialdrücken, bei denen die Elektronenleitfähigkeit den Ladungstransport dominiert, sind die statischen Abweichungen des Zellstroms deutlich niedriger als Werte, die aus der Literatur von YSZ bekannt sind. Diese statischen Abweichungen des Zellstroms lassen sich mit vergleichsweise hoher Genauigkeit quantifizieren und daher bei präziser Temperaturmessung und -regelung rechnerisch korrigieren.
Die Zeitspanne, die für die Einstellung stationärer Bedingungen nach einem Potentialsprung benötigt wird, kann mehrere Stunden betragen.

Der nach einem Potentialsprung vom Sensor freigesetzte oder aufgenommene Sauerstoff bedingt eine Ladungsmenge, die als dynamischer Messfehler einzuordnen ist. Dieser Ladungstransfer kann Werte annehmen, welche die in vielen Applikationen angestrebte Auflösung solcher Sensoren um mehrere Zehnerpotenzen übersteigt.

Die konsequente Berücksichtigung und Minimierung der bei coulometrischen Festelektrolytsensoren auftretenden materialbedingten $\mathrm{Ab}$ weichungen vom Faradayschen Gesetz ermöglicht deren Applikation für Konzentrationsmessungen im unteren ppb-Bereich, wie Untersuchungen an entsprechenden Detektoren für die Gaschromatographie belegen.

\section{Danksagung}

Das dieser Arbeit zugrunde liegende Vorhaben wird mit Mitteln des Bundesministeriums für Umwelt, Naturschutz und Reaktorsicherheit (BMU) unter dem Förderkennzeichen 03KB067B gefördert. Die Verantwortung für den Inhalt dieser Arbeit liegt bei den Autoren, die für die Förderung danken.

\section{Literatur}

[1] V. Vashook, J. Zosel, U. Guth, Oxygen solid electrolyte coulometry (OSEC), J. Solid State Electrochem. 16, 3401-3421 (2012); doi: 10.1007/s10008-012-1876-3

[2] J. Maier, Festkörper - Fehler und Funktion, B. G.Teubner, Leipzig, 2000

[3] J.-H. Park, N. Blumenthal, Electronic Transport in 8 Mole Percent $\mathrm{Y}_{2} \mathrm{O}_{3}-\mathrm{ZrO}_{2}$, J. Electrochem. Soc. 136, 2867-2876 (1989); doi: 10.1149/1.2096302

[4] M. Schelter, J. Zosel, W. Oelßner, U. Guth, M. Mertig, A solid electrolyte sensor for trace gas analysis, Sensor. Actuat. B 187 209-214 (2013); doi: 10.1016/j.snb.2012.10.111

[5] R. Hartung, Bestimmung der Elektronenteilleitfähigkeit des Festelektrolyten $\mathrm{Zr}_{0,82} \mathrm{Y}_{0,10} \mathrm{Mg}_{0,08} \mathrm{O}_{1,87}$ nach der Hebb-Wagnerschen Polarisationsmethode, Z. phys. Chem., Leip. 254, 393-410 (1973)

[6] M. Schelter, Entwicklung eines Festelektrolytsensor-Messsystems für die coulometrische Spurenanalytik, Dissertation, Technische Universität Dresden (2015); urn: nbn:de:bsz:14-qucosa-178006

[7] S. B. Adler, Factors Governing Oxygen Reduction in Solid Oxide Fuel Cell Cathodes, Chem. Rev. 104, 4791-4844 (2004); doi: $10.1021 / \mathrm{cr} 0207240$

[8] H. Rickert, Electrochemistry of Solids - An Introduction, Springer, Berlin, 1982 